1 Universidade Federal da Paraíba (UFPB),

Departamento de Clínica e Odontologia Social - João Pessoa (PB), Brasil.

chsmfreitas@hotmail.com

2 Universidade Estadual de Campinas (Unicamp), Instituto de Biologia Campinas (SP), Brasil. lemos.george@yahoo.com.br

3 Universidade Federal da Paraíba (UFPB)

Departamento de Clínica e Odontologia Social - João Pessoa (PB), Brasil. talitha.ribeiro@yahoo.com.br

4 Secretaria de Estado da Saúde da Paraíba - João Pessoa (PB), Brasil. macaiba59@hotmail.com

5 Universidade Federal da Paraíba (UFPB), Departamento de Clínica e Odontologia Social - João Pessoa (PB), Brasil. fdsforte@terra.com.br

\section{Atenção em saúde bucal: avaliação dos centros de especialidades odontológicas da Paraíba}

\author{
Attention to oral health: evaluation of the dental specialty centers in \\ Paraíba
}

Cláudia Helena Soares de Morais Freitas', George Azevedo Lemos ${ }^{2}$, Talitha Rodrigues Ribeiro

Fernandes Pessoa ${ }^{3}$, Marcílio Ferreira de Araujo ${ }^{\mathbf{4}}$, Franklin Delano Soares Forte ${ }^{\mathbf{5}}$

RESUMO Este estudo objetivou avaliar a Política Nacional de Saúde Bucal na atenção de média complexidade, considerando o desempenho dos Centros de Especialidades Odontológicas (CEOs) da Paraíba. Foram avaliados 19 CEOs, utilizando-se dados secundários da produção de 2007 a 2010, registrados pelo Sistema de Informação Ambulatorial do Sistema Único de Saúde (SIA/SUS). Os dados foram obtidos, tabulados e organizados de acordo com os subgrupos de procedimentos odontológicos, segundo a Portaria Ministério da Saúde/Gabinete do Ministro $\mathrm{n}^{\circ}$ 600. O desempenho insatisfatório dos CEOs é revelador de falhas, especialmente na gestão e na organização desses serviços. O processo de avaliação é uma etapa fundamental para garantir uma melhor qualidade dos serviços à população.

PALAVRAS-CHAVE Saúde bucal; Avaliação de serviços de saúde; Especialidades odontológicas.

ABSTRACT This study aimed to evaluate the National Policy of Oral Health in the attention of medium complexity, considering the performance of Dental Specialty Centers (DSC) in the state of Paraíba. Were evaluated 19 DSCs, using secondary data of the production from 2007 to 2010, recorded by the Outpatient Information System of the Unified Health System (SIA/SUS). Data were collected, tabulated, and organized according to subgroups of dental procedures, according to the Decree Ministry of Health/Office of the Minister, $n^{\circ}$ 600. The poor performance of the DSCs is fault revealing, especially in the management and in the organization of these services. The evaluation process is a critical step to ensure better quality of services to the population.

KEYWORDS Oral health; Health services evaluation; Specialties, Dental. 


\section{Introdução}

A população brasileira enfrenta muitos problemas de acesso aos serviços públicos de saúde, nos quais também se enquadram os serviços públicos de saúde bucal. Os resultados do levantamento das condições de saúde bucal da população brasileira - SB Brasil 2003 (BRASIL, 2004C) - comprovaram a desigualdade relacionada ao acesso aos serviços odontológicos, a gravidade e a precocidade de perda dentária, e que o edentulismo se constitui como um problema de saúde pública persistente no Brasil. A necessidade de uso de próteses já é identificada em adolescentes, principalmente nas regiões Norte e Nordeste. A população, em todos os grupos etários investigados, apresentou maiores necessidades de restaurações, tratamentos pulpares e exodontias.

Narvai et al. (2006), com dados de uma revisão de artigos e documentos produzidos no Brasil, no período de 1980 a 2003, sobre a experiência de cárie em dentes permanentes de escolares na idade de 12 anos, observaram uma tendência consistente de queda no índice de dentes cariados, perdidos e restaurados (CPOD) ao longo do período, que corresponde a um declínio de $61,7 \%$. Apesar dos inegáveis avanços no declínio do CPOD, ainda persiste um quadro de iniquidade na distribuição da cárie, o que pode ser explicado pelas precárias condições de existência a que é submetida uma grande parte da população, configurando um quadro de 'apartheid social'.

Em se tratando de atenção secundária e terciária, dados do Sistema de Informação Ambulatorial do Sistema Único de Saúde (SIA/SUS) de 2003 indicaram que os serviços especializados correspondiam a não mais que $3,5 \%$ do total de procedimentos odontológicos (BRASIL, 2006C), evidenciando a grande desproporção na oferta entre procedimentos odontológicos básicos e especializados.

Baseado no perfil epidemiológico traçado pelo SB Brasil 2003, o Ministério da Saúde (MS) lançou, em 2004, a Política Nacional de Saúde Bucal (PNSB) expressa no Brasil Sorridente, que se constitui como um conjunto de ações que busca assegurar a integralidade na atenção odontológica. As diretrizes desta política apontam para a reorganização da atenção à saúde bucal em todos os níveis de atenção, com desenvolvimento de ações intersetoriais, tendo o conceito do cuidado como eixo de reorientação do modelo (BRASIL, 2004C).

Uma das estratégias para ampliar o acesso e qualificar a oferta de serviços na atenção de média complexidade foi a criação dos Centros de Especialidades Odontológicas (CEOs). Nesse sentido, a Portaria Ministério da Saúde/Gabinete do Ministro (MS/GM) n ${ }^{0}$ 1.570 (BRASIL, 2004A) instituiu critérios, normas e requisitos para a implantação e o credenciamento dos CEOs, e a Portaria MS/GM n ${ }^{0} 1.571$ (BRASIL, 2004B) estabeleceu o seu financiamento. Posteriormente, essas foram substituídas, respectivamente, pelas Portarias MS/GM n ${ }^{\circ} 599$ (BRASIL, 2006A) e MS/GM n ${ }^{0} 600$ (BRASIL, 2006B).

Os CEOs são unidades de saúde destinadas ao atendimento odontológico especializado no âmbito do SUS, servindo como referência para a atenção primária. Estes centros participam do Cadastro Nacional de Estabelecimentos de Saúde (CNES) e ofertam, no mínimo, as seguintes especialidades: diagnóstico bucal, com ênfase no diagnóstico precoce e na detecção do câncer bucal; procedimentos periodontais e endodônticos; cirurgia oral menor de tecidos moles e duros; e atendimento a pessoas com necessidades especiais (BRASIL, 2006A).

A implantação dos CEOs constitui uma estratégia relevante com vistas à integralidade da atenção no âmbito odontológico (CHAVES ET AL., 2010) e, como serviços de saúde que representam, devem ser avaliados, objetivando a melhoria contínua da qualidade do serviço ofertado (LIMA ET AL., 2010).

O processo de avaliação e monitoramento é uma etapa fundamental para a implementação do modelo de atenção. A avaliação de políticas e programas é elemento essencial para 
o planejamento, a redefinição de prioridades e no redirecionamento de ações, sendo prioritário que a PNSB implantada em 2004 seja avaliada, pois pouco se sabe sobre os avanços, as dificuldades e o impacto desta sobre a saúde da população.

A avaliação de como vem sendo feita a oferta da atenção nos CEOs poderá elucidar avanços da PNSB nos serviços de média complexidade, assim como reorientar a construção dos mesmos, pela identificação dos problemas que emergem das vivências e da prática. A avaliação da atenção secundária em saúde bucal prestada pelo SUS no estado do Nordeste brasileiro cenário da presente pesquisa, até bem pouco tempo, seria difícil, visto que os locais de prestação de serviços não existiam ou eram escassos. Nos anos 2000, após a definiç̧ão da PNSB (2004C), houve um investimento por parte do governo brasileiro na criação desses serviços. Assim, torna-se necessária a avaliação desse cenário como prestador de serviços à população brasileira.

Dessa forma, o objetivo do estudo foi avaliar a PNSB na atenção de média complexidade, considerando o desempenho e a efetividade dos CEOs em um estado do Nordeste do Brasil, na busca da integralidade do cuidado e da qualidade da atenção à saúde.

\section{Material e métodos}

Trata-se de um estudo transversal, quantitativo, de caráter avaliativo normativo, da integralidade do cuidado em saúde bucal na atenção de média complexidade da Paraíba, no período de 2007 a 2010.

Segundo informações do MS (BRASIL, 2009), foram identificados, até dezembro de 2008, na Paraíba, 31 CEOs habilitados, os quais constituíram o universo da pesquisa. Entretanto, apesar de habilitados, não constava registro da produção ambulatorial de todos os municípios no SIA/SUS. Para seleção dos municípios e CEOs neste estudo foram adotados os seguintes critérios de inclusão: municípios com
CEOs cuja portaria de habilitação ministerial tenha sido publicada em período igual ou superior a dois anos; e CEOs com período de funcionamento igual ou superior a dois anos, a partir do registro de sua produção ambulatorial pelo SIA/SUS, de forma a se analisar uma série histórica. A amostra final do estudo foi composta de 19 municípios, com apenas 1 CEO em cada município, para o período de análise de 2008 a 2010. E no período de análise de 2007 a 2010, a amostra foi composta por 16 municípios, com apenas 1 CEO. Os municípios foram agrupados em quatro estratos populacionais, conforme tabela 1.

Tabela 1. Municípios estudados com CEO de acordo com estrato populacional IBGE, 2008, Paraíba/Brasil

\begin{tabular}{|c|c|}
\hline Município & População \\
\hline \multicolumn{2}{|c|}{ Até 20 mil habitantes } \\
\hline A & 14.715 \\
\hline B & 15.236 \\
\hline C & 15.881 \\
\hline D & 17.173 \\
\hline E & 17.496 \\
\hline \multicolumn{2}{|c|}{ De 21 a 40 mil habitantes } \\
\hline $\mathrm{F}$ & 23.023 \\
\hline G & 24.752 \\
\hline $\mathrm{H}$ & 26.279 \\
\hline । & 27.548 \\
\hline J & 29.980 \\
\hline $\mathrm{L}$ & 31.524 \\
\hline \multicolumn{2}{|c|}{ De 41 a 80 mil habitantes } \\
\hline M & 41.283 \\
\hline N & 54.200 \\
\hline O & 63.783 \\
\hline \multicolumn{2}{|c|}{ Acima de 81 mil habitantes } \\
\hline P & 92.891 \\
\hline Q & 97.276 \\
\hline $\mathrm{R}$ & 122.454 \\
\hline S & 371.060 \\
\hline T & 674.762 \\
\hline
\end{tabular}


Para a avaliação do desempenho dos CEOs, utilizaram-se dados secundários da produção ambulatorial, a partir da série histórica da produção de procedimentos odontológicos (quantidade apresentada) realizados pelos CEOs, registrados pelo SIA/ SUS no período de 2007 a 2010. A coleta dos dados foi realizada diretamente no banco de dados do Departamento de Informática do Sistema Único de Saúde (Datasus), informações do SIA/SUS, segundo o cadastro nacional de estabelecimento de saúde dos CEOs avaliados.

Os dados foram obtidos e tabulados pelo programa Tab para Windows (Tabwin), exportados para o programa Excel, versão 2007, onde foi feita a consolidação e o agrupamento dos dados, de acordo com os subgrupos de procedimentos odontológicos e segundo a Portaria MS/GM n ${ }^{\circ} 600$ (BRASIL, 2006B). Dessa forma, os procedimentos realizados foram agrupados em quatro subgrupos: periodontia; endodontia; cirurgia; e procedimentos básicos (para atendimento a pacientes com necessidades especiais).

A meta anual de cada subgrupo foi obtida a partir da soma das metas mensais dos respectivos subgrupos, estabelecidas na Portaria MS/GM n ${ }^{\circ} 600$ (BRASIL, 2006B). Em seguida, comparou-se a quantidade anual de procedimentos realizados pelos CEOs com as metas anuais em cada subgrupo de especialidade. Considerou-se meta atingida o cumprimento de percentual igual ou superior a $100 \%$ da meta anual preconizada para cada subgrupo de procedimentos. A partir desses dados, classificou-se o desempenho dos serviços em: desempenho ruim (CEOs que cumpriram a meta em até uma especialidade); desempenho regular (cumprimento das metas em duas especialidades); desempenho bom (cumprimento das metas em três especialidades); e desempenho ótimo (CEOs que cumpriram as metas em todas as especialidades). Para os meses ausentes no Datasus, os subgrupos foram considerados zero (0).
Para fins de comparação, foram coletados no SIA/SUS os dados referentes aos procedimentos de média complexidade em odontologia, no período anterior ao lançamento das diretrizes da PNSB (2000 A 2004) pelo MS.

Como variáveis independentes foram consideradas: porte populacional; Índice de Desenvolvimento Humano (IDH); cobertura de Equipe de Saúde da Família (EqSF); e cobertura de Equipe Saúde Bucal (EqSB), nos anos de 2007, 2008, 2009 e 2010. A população dos municípios estudados foi obtida a partir do Instituto Brasileiro de Geografia e Estatística (IBGE). O IDH foi obtido a partir de dados do IBGE, sendo também considerada a classificação do Programa das Nações Unidas para o Desenvolvimento (PNUD).

As coberturas de EqSF e EqSB foram obtidas a partir de dados do CNES, considerando-se a população e o número de EqSF e EqSB implantadas. Como as coberturas de EqSF e EqSB não apresentaram diferenças significativas, foram considerados, no estudo, apenas os percentuais obtidos no ano de 2008, primeiro a conter todos os 19 CEOs devidamente habilitados.

O presente estudo foi devidamente registrado nas bases de pesquisa da Universidade Federal da Paraíba, submetido e aprovado pelo Comitê de Ética em Pesquisa (CEP) da instituição responsável, certidão CEP/ Hospital Universitário Lauro Wanderley $n^{0} 198 / 2010$, seguindo as orientações das normas previstas na legislação nacional.

\section{Resultados}

Para a apresentação dos dados, os CEOs investigados receberam um código em forma de letra. Foram estudados 16 CEOs no ano de 2007 e 19 CEOs nos anos de 2008, 2009 e 2010, sendo a maioria do tipo I (50\%) em 2007 e do tipo II (52,6\%) em 2008, 2009 e 2010. 
A análise do cumprimento das metas, segundo o tipo de CEO, apontou que, dentre os CEOs tipo I, nenhum teve desempenho ótimo nos anos de 2007, 2008 e 2009. No entanto, no ano de 2010, apenas um CEO tipo
I atingiu tal desempenho. Nenhum CEO tipo II apresentou desempenho ótimo nos quatro anos analisados. Já o CEO tipo III exibiu um desempenho bom no ano de 2007 e ótimo em 2008, 2009 e 2010 (tabela 2).

Tabela 2. Relação entre as características avaliativas dos serviços e dos municípios, segundo o desempenho dos CEO, Paraíba/Brasil

\begin{tabular}{|c|c|c|c|c|c|c|c|c|}
\hline \multirow{2}{*}{ Município } & \multirow{2}{*}{$\begin{array}{c}\text { Tipo de } \\
\text { CEO }\end{array}$} & \multirow{2}{*}{$\begin{array}{c}\text { Cobertura } \\
\text { EqSF } 2008 \\
(\%)\end{array}$} & \multirow{2}{*}{$\begin{array}{c}\text { Cobertura } \\
\text { EqSB } \\
2008(\%)\end{array}$} & \multirow{2}{*}{ IDH } & \multicolumn{4}{|c|}{ Desempenhoa } \\
\hline & & & & & 2007 & 2008 & 2009 & 2010 \\
\hline \multicolumn{9}{|c|}{ Até 20 mil habitantes } \\
\hline A & 2 & 100 & 100 & 0,658 & $b$ & Ruim & Ruim & Ruim \\
\hline B & 1 & 100 & 100 & 0,595 & Ruim & Ruim & Ruim & Ruim \\
\hline C & 2 & 100 & 100 & 0,634 & Ruim & Ruim & Ruim & Ruim \\
\hline D & 1 & 100 & 100 & 0,595 & Ruim & Ruim & Regular & Ótimo \\
\hline$E$ & 1 & 100 & 100 & 0,608 & Bom & Regular & Ruim & Ruim \\
\hline \multicolumn{9}{|c|}{ De 21 a 40 mil habitantes } \\
\hline $\mathrm{F}$ & 1 & 100 & 100 & 0,603 & Regular & Ruim & Bom & Ruim \\
\hline G & 2 & 100 & 100 & 0,612 & Ruim & Ruim & Ruim & Ruim \\
\hline $\mathrm{H}$ & 1 & 100 & 100 & 0,568 & Regular & Ruim & Ruim & Regular \\
\hline । & 2 & 95,43 & 95,43 & 0,668 & $b$ & Bom & Ruim & Ruim \\
\hline J & 2 & 89,74 & 89,74 & 0,603 & Ruim & Ruim & Regular & Bom \\
\hline L & 1 & 83,23 & 64,77 & 0,661 & Regular & Regular & Regular & Ruim \\
\hline \multicolumn{9}{|c|}{ De 41 a 80 mil habitantes } \\
\hline M & 1 & 100 & 100 & 0,581 & Ruim & Bom & Ruim & Ruim \\
\hline N & 1 & 96,94 & 80,78 & 0,659 & Bom & Bom & Bom & Ruim \\
\hline $\mathrm{O}$ & 2 & 100 & 100 & 0,658 & Bom & Regular & Ruim & Ruim \\
\hline \multicolumn{9}{|c|}{ Acima de 81 mil habitantes } \\
\hline$P$ & 2 & 87,98 & 87,98 & 0,689 & $b$ & Ruim & Ruim & Regular \\
\hline Q & 2 & 100 & 100 & 0,678 & Ruim & Regular & Regular & Regular \\
\hline $\mathrm{R}$ & 2 & 85,81 & 85,81 & 0,659 & Regular & Bom & Ruim & Regular \\
\hline S & 2 & 69,21 & 27,53 & 0,721 & Bom & Ruim & Regular & Ruim \\
\hline T & 3 & 77,04 & 77,04 & 0,783 & Bom & Ótimo & Ótimo & Ótimo \\
\hline
\end{tabular}

Notas: a Ruim (CEOs que cumpriram a meta em até 1 especialidade); regular (CEOs que cumpriram as metas em 2 especialidades); bom (CEOs que cumpriram as metas de 3 especialidades); e, ótimo (CEOs que cumpriram as metas de todas as especialidades).

b Município com implantação do CEO apenas em 2008.

Verifica-se, no gráfico 1 , que os CEOs estudados obtiveram desempenho insatisfatório para os quatro anos analisados. Em 2007, 43,8\% dos CEOs obtiveram desempenho ruim; $52,6 \%$ em 2008; 57,9\% em 2009; e $63,2 \%$ em 2010. Nenhum CEO obteve desempenho ótimo no ano de 2007, apenas um CEO (5,3\%) obteve desempenho ótimo nos anos de 2008 e 2009, e dois CEOs em 2010 $(10,6 \%)$. 
Gráfico 1. Desempenho dos Centros de Especialidade Odontológicas por cumprimento das metas, Paraíba/Brasil

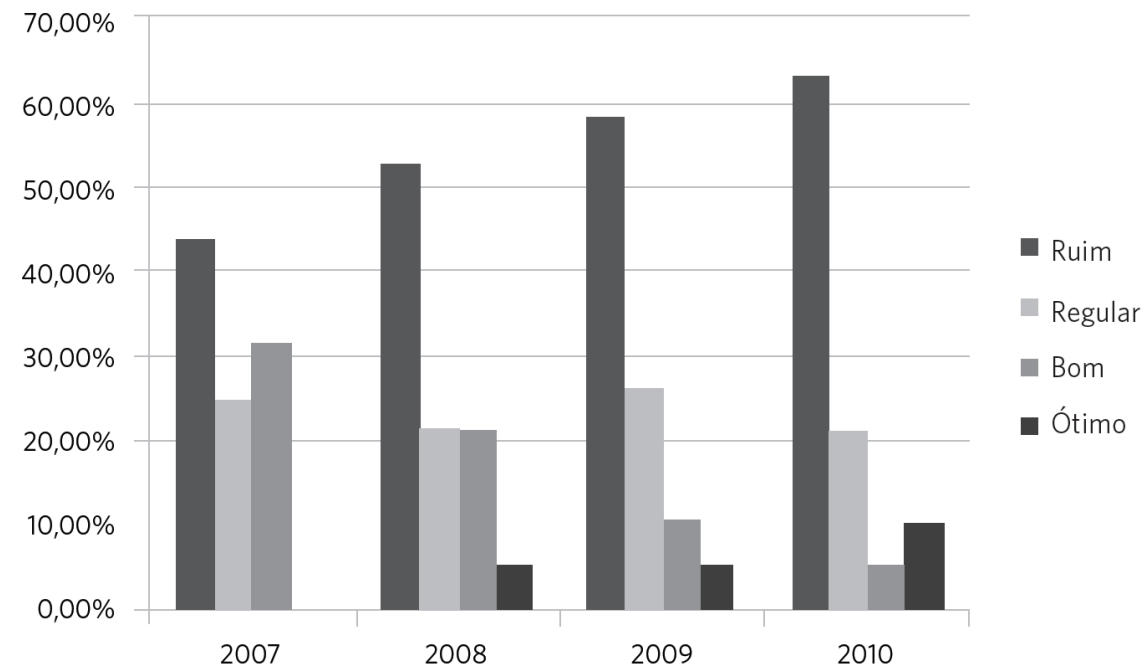

Em relação ao porte populacional, os CEOs estudados encontram-se bem distribuídos nos quatro extratos populacionais, para os quatro anos analisados, respeitando, portanto, o princípio da equidade em saúde proposto pela PNSB. Levando em consideração a cobertura municipal de EqSF e de EqSB, a maioria dos municípios apresentou percentual de cobertura entre $76 \%$ e $100 \%$. Tal fato não influenciou os resultados encontrados. O IDH de todos os municípios analisadas, de acordo com o PNUD, foi classificado como médio, com valores entre 0,500 e 0,799 para todos os anos estudados (tabela 2).

Considerando os resultados por subgrupo de especialidades, o subgrupo endodontia apresentou o pior desempenho entre as quatro especialidades analisadas nos anos de 2008, 2009 e 2010. Apenas 10,5\% dos CEOs conseguiram cumprir a meta anual preconizada de endodontia nesses três anos. No entanto, no ano de 2007, a especialidade de endodontia obteve o melhor desempenho, com $62,5 \%$ dos CEOS conseguindo cumprir as metas preconizadas. O subgrupo de procedimentos básicos obteve os melhores desempenhos nos anos de 2008 e 2009 - 68,4\% e $73,7 \%$, respectivamente -, mas obteve o pior desempenho em 2007 (25\%). Em 2010, o melhor desempenho foi observado na especialidade de periodontia (52,6\%).

Os CEOs investigados exibiram também alta produção de procedimentos básicos não regulamentados pela Portaria MS/ GM no 600 (restauração com amálgama, restauração com ionômero de vidro, restauração a pino, acesso a polpa dentária e medicação, curativo de demora etc.), sendo que os CEOs tipo I apresentaram os maiores valores em relação aos demais. Muitos desses centros de especialidades odontológicas não cumpriram as metas preconizadas, mas produziram grande quantidade de procedimentos que não são de competência da atenção secundária.

Verificou-se também que alguns dos CEOs investigados produziram procedimentos de média complexidade que não são determinados pela portaria 600/2006 (apicectomia com/sem obturação retrógrada, manutenção/conserto de aparelhos ortodônticos, colocação de mantenedor de espaço, implantes dentários etc.), mas em quantidades pequenas, com exceção de um 
município de grande porte (acima de $80 \mathrm{mil}$ habitantes), onde foram realizados 651, 338 e 243 procedimentos nos anos de 2008, 2009 e 2010, respectivamente.

Ao avaliar a produção de procedimentos especializados em odontologia no período anterior à implantação da PNSB (2000, 2001, 2002, 2003 e 2004), e após esta política (2008, 2009 e 2010), observou-se que dez (52,6\%) dos municípios investigados não ofertavam nenhum procedimento especializado neste período, e que oito $(42,1 \%)$ dos municípios apresentaram aumento considerável de oferta de procedimentos especializados à população, com exceção do município $\mathrm{S}$, conforme se observa na figura 1 .

Figura 1. Procedimentos especializados (dentística, periodontia, endodontia e cirurgia) realizados pelos 19 municípios no período anterior e após a implantação da Política Nacional de Saúde Bucal
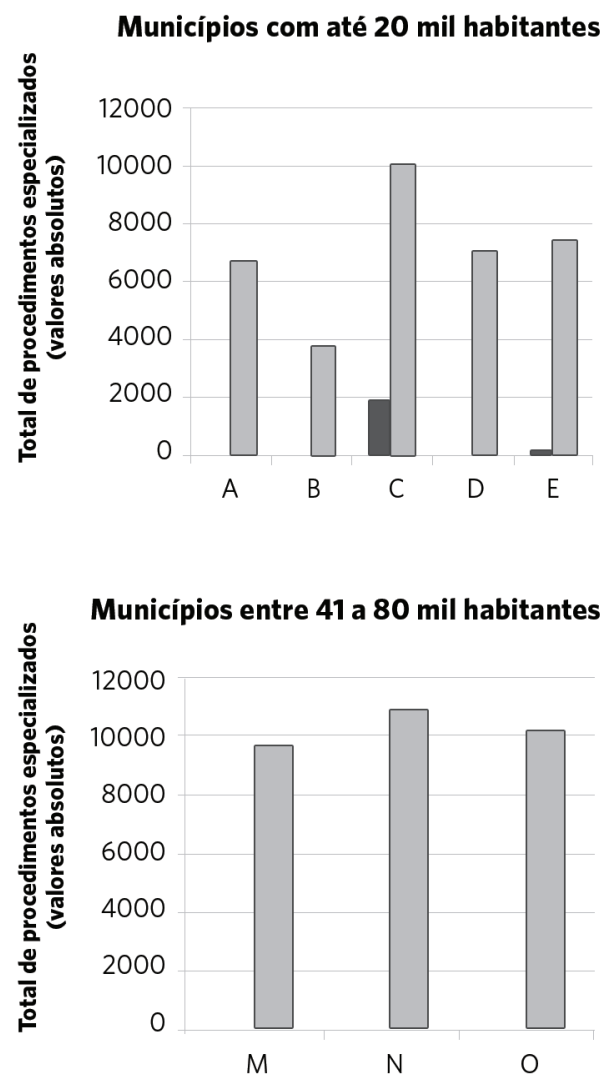

\section{Discussão}

A viabilização de uma nova prática em saúde bucal para a dignificação da vida e da conquista da cidadania depende do desenvolvimento de um modelo de atenção em

\section{Municípios entre 21 a 40 mil habitantes}
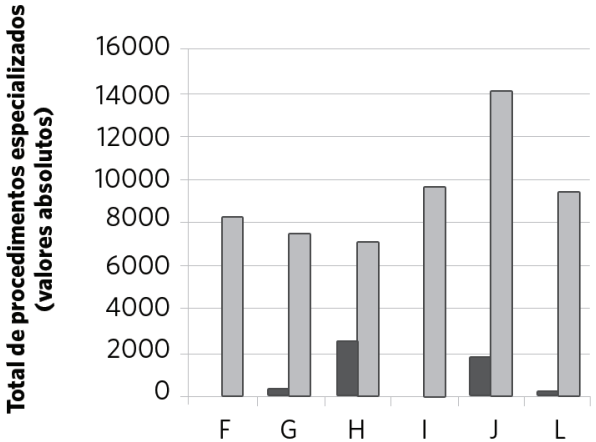

Municipios acima de $\mathbf{8 1}$ mil habitantes

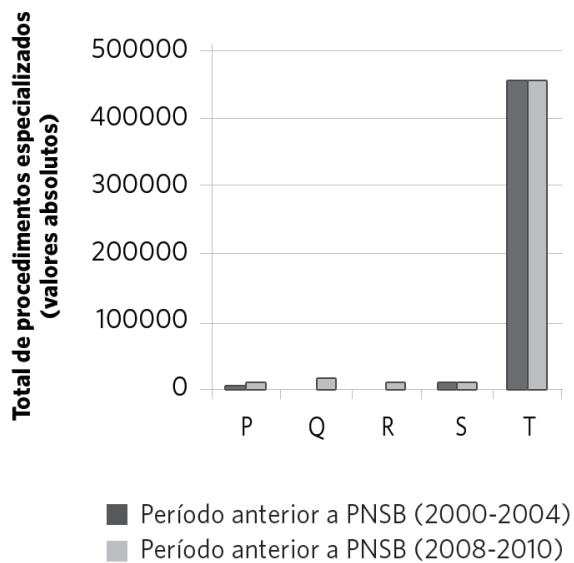

Período anterior a PNSB (2008-2010)

saúde bucal orientado pelos princípios da universalidade do acesso, da integralidade e da equidade, caracterizado pela resolubilidade das ações que realiza (BRASIL, 2004C). Nesse sentido, um dos grandes pilares norteadores da PNSB, lançada em 2004, foi a 
implantação dos CEOs, com o objetivo de garantir o acesso a procedimentos odontológicos de maior densidade tecnológica, na busca pela efetivação da integralidade da atenção.

Algumas pesquisas de avaliação da política nacional de saúde estão sendo desenvolvidas, destacando-se a média complexidade, representada pela implantação dos CEOs. Figueiredo e Goes (2009), em estudo realizado no estado de Pernambuco, observaram que $40,9 \%$ dos CEOs pesquisados obtiveram desempenho bom, salientando que o melhor cumprimento de metas foi obtido pelo subgrupo Atenção Básica (AB), em relação aos demais, principalmente à cirurgia oral menor. Porém, esses autores observaram que $31,8 \%$ dos CEOs exibiram desempenho ruim no cumprimento global das metas.

No presente estudo, verificou-se que a maioria dos CEOs não conseguiu cumprir as metas estabelecidas pela Portaria MS/GM $\mathrm{n}^{\circ} 600$, que institui o financiamento e o monitoramento dos CEOs. Destacamos o ano de 2010, que exibiu os piores resultados 63,2\% dos CEOs apresentaram desempenho ruim. Infere-se que isso possa ter acontecido porque 2010 tratou-se de um ano eleitoral e, embora o pleito tenha sido em nível estadual e federal, pode ter havido interferência no setor público municipal. Neste sentido, muitos municípios optam ainda pela realização de contratos temporários e terceirizações da mão de obra dos profissionais em saúde, resultando em precarização das relações de trabalho, insegurança e interferência política em suas atividades (SOARES; PAIM, 2011), justificando o desempenho insatisfatório observado nesse ano.

Entretanto, o desempenho dos CEOs investigados nos anos de 2007, 2008 e 2009 também foi muito insatisfatório, apresentando, respectivamente, $43,8 \%, 52,6 \%$ e $57,9 \%$ dos CEOs com desempenho ruim. Chaves et al. (2011), em estudo realizado no estado da Bahia, também demonstraram baixa taxa de utilização dos serviços públicos odontológicos especializados, conforme os padrões propostos pela Portaria $n^{0}$ $1.101 / G M$, de 2002 , e pela consulta aos especialistas, ou seja, a oferta disponível está, de fato, sendo subutilizada para um tipo de serviço essencial à garantia da integralidade da atenção à saúde bucal. Estudos de Cortellazzi et al. (2014) verificaram que, em relação ao cumprimento global das metas, a maioria dos CEOs - cerca de 69,25\% - apresentou desempenho ruim/regular.

Em relação aos subgrupos de especialidades, chama a atenção o baixo desempenho da endodontia nos anos de 2008, 2009 e 2010. Apenas 10,5\% dos CEOs conseguiram cumprir a meta anual preconizada de endodontia nesses três anos, revelando uma subutilização do serviço. Lino et al. (2014) verificaram, em Minas Gerais, que em 77\% dos municípios estudados a taxa de procedimento do subgrupo endodontia foi igual a zero (0) para cada 10 mil habitantes, no ano de 2010. Sousa e Chaves (2010) também observaram baixa taxa de utilização dos procedimentos de endodontia, representando apenas $16,1 \%$. Para estes autores, a relação entre o que deveria ser ofertado e o que está sendo utilizado está muito abaixo do padrão desejável de bom desempenho dos serviços de saúde e pode ser explicada pela falta de um sistema de gestão de metas de produção por especialidade.

Santana et al. (2008), em estudo realizado na cidade de Recife (PE), também corroboram o diagnóstico de insuficiência de serviços especializados e a dificuldade de acesso aos mesmos, principalmente na especialidade de endodontia. Estes autores também observam que, na tentativa de reorganização do modelo de atenção à saúde bucal, este município tenta imprimir às Unidades Básicas de Saúde (UBS) a responsabilidade pelo atendimento da média complexidade, oferecendo alguns serviços especializados, desvirtuando a lógica de funcionamento da unidade básica. Já o estudo realizado por Cortellazzi et al. (2014) demonstrou que 
68,22\% dos CEOs cumpriram as metas na $\mathrm{AB}, 55,94 \%$ de periodontia e menores percentuais para a cirurgia $33,07 \%$, e endodontia $22,61 \%$.

No entanto, Chaves et al. (2010), em um estudo realizado em quatro municípios no estado da Bahia, verificaram que os usuários que necessitavam de tratamento endodôntico tiveram 2,62 vezes mais chances de concluir o tratamento do que aqueles que procuravam os centros de especialidade para tratar lesões de mucosa ou realizar cirurgia oral menor, divergindo dos nossos resultados.

A literatura tem demonstrado a existência de uma grande demanda reprimida na $\mathrm{AB}$, em relação à especialidade endodontia (MEDEIROS, 2007; LINO ET AL., 2014). Neste sentido, as longas filas e a demora no atendimento acabam contribuindo para o elevado número de abandonos de tratamento pelos usuários. Além disso, o descrédito quanto aos tratamentos conservadores reforça a banalização das exodontias e a consequente rejeição ao tratamento endodôntico. Estes fatores justificam o baixo desempenho desta especialidade observado no presente estudo.

Observou-se também que a maioria dos municípios possui uma ampla rede de atenção primária, com percentuais de cobertura de EqSF e EqSB entre $76 \%$ e $100 \%$, mas uma rede de atenção secundária em odontologia com desempenho insatisfatório. Fatores como desarticulação da rede, falhas no sistema de referência e contrarreferência, e precarização do vínculo usuário/ profissional devem ser analisados, pois podem prejudicar o acesso aos serviços de média complexidade em odontologia, causando grande demanda reprimida.

Conflitos importantes podem ser observados na integração entre $\mathrm{AB}$ e a atenção especializada, não apenas na referência adequada de casos para este nível de atenção, mas também na chegada do usuário ao CEO sem a devida adequação do meio bucal e promoção de saúde, funções da atenção primária (CHAVES ET AL., 2011). Estes fatores podem estar contribuindo para o desempenho insatisfatório dos CEOs observado neste estudo.

$A$ interface ideal entre os serviços de $A B$ e a secundária deve levar em consideração as seguintes características: equidade, integralidade e eficiência, para garantir acesso, referência e contrarreferência adequados (FIGUEIREDO; GOES, 2009). Uma rede de atenção primária estruturada e bem articulada, capaz de operacionalizar a referência e contrarreferência de usuários, é indispensável para a garantia da integralidade na assistência à saúde bucal. Além disso, não é recomendada a implantação de CEOs em municípios onde a $\mathrm{AB}$ não está adequadamente estruturada. A atenção secundária estaria exposta às pressões da livre demanda e à execução de procedimentos típicos de atenção primária, desviando-se do seu objetivo central, que é garantir a integralidade na saúde bucal ao oferecer procedimentos de maior densidade tecnológica (CHAVES ET AL., 2010).

Em nosso estudo, foi verificada uma produção acentuada de procedimentos básicos pela maioria dos CEOs, que não são de competência deste nível de atenção. Essa situação também foi identificada no estudo de Figueiredo e Goes (2009). São necessários mais estudos relacionados à organização e à articulação da rede de $\mathrm{AB}$ no estado para explicar melhor tais hipóteses.

Verificou-se também que alguns CEOs avaliados produziram procedimentos de média complexidade não regulamentados pela Portaria MS/GM no 600. Os valores foram muito pequenos, à exceção de um município de grande porte, que exibiu uma produção de 651, 338 e 243 procedimentos, para os anos de 2008, 2009 e 2010, respectivamente. O CEO desse município encontrava-se instalado em uma instituição de ensino superior em odontologia, onde possivelmente eram realizados outros procedimentos de média complexidade, por fazerem parte da formação prática dos 
alunos dessa instituição.

O baixo desempenho em algumas especialidades desenvolvidas nos CEOs pode ser revelador de problemas de gestão do serviço, destacando-se a ausência de clareza sobre os padrões e metas propostos pelo serviço e a constante falta de usuários, que não são substituídos, além das diferentes tecnologias utilizadas por cada especialista, o que dificulta possíveis padronizações, a exemplo da endodontia, em que alguns especialistas optam por tratamentos longos e completos por sessão (abertura e fechamento), enquanto outros elegem o modelo de tratamentos curtos e mais fracionados (CHAVES ET AL., 2011).

A falta de conhecimento do serviço ou indicação correta pela $\mathrm{AB}$ também pode levar à menor procura pelos serviços especializados e explicar o desempenho insatisfatório dos CEOs observado no estudo. Por outro lado, o número de profissionais por especialidades nos centros pode ser insuficiente, sugerindo que, no âmbito do SUS, a expansão da rede assistencial de atenção odontológica secundária não acompanhou o crescimento da oferta de serviços da $A B$ (LIMA ET AL., 2010).

Além disso, o descumprimento das metas estabelecidas pode sugerir dificuldades com a permanência dos recursos humanos nos CEOs, principalmente em cidades de menor porte. Cabe destacar, inclusive, que os profissionais lotados na atenção pública especializada podem ter dupla militância, o que, embora a atuação profissional no serviço público e o exercício privado da odontologia não sejam conflitantes, é um desafio para os gestores públicos na medida em que a lógica dominante do campo privado odontológico está presente nas aspirações dos profissionais, que não se consideram muitas vezes ajustados ou incorporados ao campo da saúde pública (CHAVES ET AL., 2011).

Santana et al. (2008) observaram aumento considerável nos procedimentos especializados em odontologia na cidade do Recife, em todos os anos analisados (2000-2007). Estes autores verificaram ainda que os procedimentos de saúde bucal realizados nos CEOs exibiram um padrão crescente, ano a ano, a partir de sua implantação, representando, em 2004, 11,71\% dos procedimentos realizados na média complexidade, aumentando, em 2007, para 29,71\%, demonstrando que os CEOs, apesar de serem parte de uma estratégia do MS para atender à demanda da média complexidade, respondem por uma quantidade minoritária de procedimentos para a população, sendo que a maioria destes continua sendo realizada por policlínicas conveniadas ao SUS.

Estudos realizados por Cortellazzi et al. (2014) verificaram que municípios pertencentes às regiões Nordeste, Sul e Sudeste tiveram prevalência de CEO com desempenho ruim/regular maior do que aqueles localizados na região Norte. Municípios com IDH médio $\leq 0,77$, com densidade demográfica inferior ou igual a 98,81 e tempo de implantação do CEO $\leq 2$ anos apresentaram prevalência maior de associação com desempenho ruim/regular do CEO. Já no estudo, em Minas Gerais, por Lino et al. (2014), foi observado que o tempo de implantação do CEO interfere na eficiência, ou seja, quanto mais tempo, mais eficiente. No presente, todos os municípios exibiram IDH médio com valores entre 0,500 e 0,799, e o IDH ou tempo de implantação não influenciaram o desempenho dos CEOs.

Ao comparar a produção de procedimentos especializados em odontologia no período anterior e no posterior à PNSB, observou-se considerável aumento dos procedimentos especializados em quase todos os municípios estudados. Foi evidente o crescimento da oferta de procedimentos especializados após a implantação da PNSB. No entanto, o desempenho insatisfatório dos CEOs observado neste estudo é revelador de falhas desta política, especialmente no campo da gestão e da organização dos serviços de especialidades odontológicas. 
As metas propostas pelas portarias ministeriais são questionáveis, visto que não se baseiam na oferta em potencial de procedimentos por especialidade, levando em conta apenas o tipo de CEO. Esta discussão é uma lacuna relevante no sentido de investigar a relação existente entre a oferta e a utilização desses serviços (CHAVES ET AL., 2010).

Figueiredo e Goes (2009) afirmaram a necessidade de adequação dos critérios e normas para implantação e monitoramento dos CEOs. Estes autores consideram essenciais novas pesquisas que abordem o uso de protocolos clínicos específicos, implantação e efetividade de protocolos de referência e contrarreferência, avaliação para qualidade da atenção e grau de satisfação de usuários. O estabelecimento de metas pelo MS é um ponto crítico, segundo Lino et al. (2014), pois as metas deveriam ser propostas a partir da capacidade de produção dos procedimentos. Assim, a produção de tratamentos endodônticos deveria ser diferenciada, de acordo com o elemento dentário envolvido. Essas metas deveriam ser revistas e consideradas em conjunto com o desempenho do município nas ações de $\mathrm{AB}$ em saúde bucal (MAIA; KORNIS, 2010).

Os critérios e normas para monitoramento dos CEOs tomam como base apenas o tipo de CEO e não o contexto socioeconômico e a dinâmica social do município onde estão inseridos, induzindo uma supervalorização do teor quantitativo em detrimento da atenção e do acolhimento humanizado ao usuário.

Esta concepção de prática centrada na assistência odontológica ao indivíduo doente e na quantidade de procedimentos ofertados revela a essência da odontologia de mercado, que ainda exerce forte influência nos serviços públicos de saúde bucal, especialmente na atenção de média complexidade. A odontologia de mercado organiza-se ao modo de produção capitalista, com a transformação dos cuidados de saúde em mercadorias. A saúde é tida como um bem comum, sem valor de troca, e sobre a qual as deformações mercantilistas e éticas sobejamente conhecidas são impostas (NARVAI, 2001).

Como principal limitação deste estudo, pode-se apontar a utilização de dados de sistemas de informação em saúde, a exemplo do SIA/SUS, tendo em vista o número acentuado de fatores que podem interferir no registro de procedimentos. No entanto, a agilidade, a rapidez na disponibilidade dos dados e a possibilidade de analisar o perfil da oferta, o sistema de informação um importante instrumento de avaliação dos serviços de saúde. Lino et al. (2014) apontaram que os sistemas de informação, em termos de gestão, facilitam a avaliação, a partir da leitura dos dados produzidos e registrados nesses sistemas.

Os sistemas de informação são importantes, não apenas do ponto de vista de financiamento, faturamento e prestação de contas, mas, sobretudo como subsídio concreto para programação e planejamento da atenção à saúde, sendo essencial a necessidade de capacitação dos profissionais e gestores no tocante à operacionalização e à análise dos bancos de dados disponíveis (MAIA; KORNIS, 2010).

\section{Conclusões}

A cobertura de EqSF e EqSB nos municípios não influenciou os resultados encontrados, assim como o IDH de todos os municípios analisados. No geral, o desempenho dos CEOs tipo I foi ruim ou regular. Já o CEO tipo III exibiu um desempenho bom no ano de 2007 e ótimo nos anos seguintes. Os CEOs paraibanos apresentaram produção de procedimentos básicos não regulamentados pela Portaria MS/GM n ${ }^{\circ} 600 / 2006$, sendo que os CEOs tipo I apresentaram maiores valores em relação aos demais.

Foi constatada uma expansão de serviços de saúde bucal no sistema de atenção secundária no estado pesquisado, o que tornou 
possível uma maior oferta de procedimentos odontológicos especializados para a população, na perspectiva de uma atenção integral. Isto demonstra a importância da implantação da PNSB, contemplando os diversos níveis de atenção. Entretanto, na perspectiva de ampliar o acesso à assistência à saúde bucal, visando ao cuidado integral, faz-se necessário fortalecer a Atenção Primária à
Saúde, para que não ocorra uma subutilização do serviço especializado. Sugere-se a realização de outros estudos de avaliação da PNSB, tanto de caráter quantitativo quanto qualitativo, a fim de elucidar avanços e dificuldades para reorientar a construção coletiva de uma política de saúde bucal que garanta o acesso, seguindo os princípios da integralidade e da equidade.

\section{Referências}

BRASIL. Ministério da Saúde. Portal da saúde. Brasília, DF: Ministério da Saúde, 2009. Disponível em: <http:// www.saude.gov.br>. Acesso em: 1 set. 2009.

Ministério da Saúde. Portaria MS/GM n ${ }^{\circ} 1.570$,

de 29 de julho de 2004. Estabelece critérios, normas e requisitos para a implantação e credenciamento de centros de especialidades odontológicas e laboratórios regionais de próteses dentárias. Diário Oficial [da] República Federativa do Brasil, Brasília, DF, 30 jul. 2004a, p. 71. Seção 1.

Ministério da Saúde. Portaria MS/GM n ${ }^{\circ}$ 1.571, de 29 de julho de 2004. Estabelece o financiamento dos Centros de Especialidades Odontológicas - CEO. Diário Oficial [da] República Federativa do Brasil, Brasília, DF, 30 jul., 2004b, p. 72, Seção 1.

Ministério da Saúde. Portaria MS/GM nº 599, de 23 de março de 2006. Define a implantação de Especialidades Odontológicas (CEO) e de Laboratórios Regionais de Próteses Dentárias (LRPDs) e estabelece critérios, normas e requisitos para seu credenciamento. Diário Oficial [da] República Federativa do Brasil, Brasília, DF, 24 mar. 2006a, p. 51, Seção 1.

Ministério da Saúde. Portaria MS/GM nº 600, de 23 de março de 2006. Institui o financiamento dos Centros de Especialidades Odontológicas. Diário Oficial [da] República Federativa do Brasil, Brasília, DF, 24 mar., 2006b, p. 52, Seção 1.
Ministério da Saúde. Secretaria de Atenção à Saúde. Departamento de Atenção Básica. Coordenação Nacional de Saúde Bucal. Diretrizes da política nacional de saúde bucal. Brasília, DF, 2004c.

Ministério da Saúde. Secretaria de Atenção à Saúde. Departamento de Atenção Básica. Coordenação Nacional de Saúde Bucal. Política Nacional de Saúde Bucal: registro de uma conquista história. Brasília, DF, $2006 c$.

CHAVES, S. C. L. et al. Avaliação da oferta e utilização de especialidades odontológicas em serviços públicos de atenção secundária na Bahia, Brasil. Cadernos de Saúde Pública, Rio de Janeiro, v. 27, n. 1, p. 143-154, 2011.

CHAVES, S. C. L. et al. Política Nacional de Saúde Bucal: fatores associados à integralidade do cuidado. Revista de Saúde Pública, São Paulo, v. 44, n. 6, p. 10051013, 2010.

CORTELLAZZI, K. L. et al. Variáveis associadas ao desempenho de Centros de Especialidades Odontológicas no Brasil. Revista Brasileira de Epidemiologia, São Paulo, v. 17, n. 4, p. 978-988, 2014.

FIGUEIREDO, N.; GOES, P. S. A. Construção da atenção secundária em saúde bucal: um estudo sobre os centros de especialidades odontológicas em Pernambuco, Brasil. Cadernos de Saúde Pública, Rio de Janeiro, v. 25, n. 2, p. 259-267, 2009. 
LIMA, A. C. S. et al. Satisfação dos usuários assistidos nos Centros de Especialidades Odontológicas do Município do Recife, Pernambuco, Brasil. Cadernos de Saúde Pública, Rio de Janeiro, v. 26, n. 5, p. 991-1002, 2010.

LINO, P. A. et al. Análise da atenção secundária em saúde bucal no estado de Minas Gerais, Brasil. Cadernos de Saúde Pública, Rio de Janeiro, v. 19, n. 9, p. 3879-3888, 2014.

MAIA, L. S.; KORNIS, G. E. M. A Reorganização da atenção à saúde bucal frente aos incentivos federais: a experiência fluminense. Revista de APS, Juiz de Fora, v. 13, n. 1, p. 84-95, 2010.

MEDEIROS, E. Os centros de especialidades odontológicas como suporte da atenção básica: uma avaliação na perspectiva da integralidade. 2007. $173 \mathrm{f}$. Dissertação (Mestrado em Odontologia) - Universidade Federal do Rio Grande do Norte, Natal, 2007.

NARVAI, P. C. Saúde bucal coletiva: um conceito. Revista do IEEE América Latina, São Paulo, v. 3, n. 1/2, p. 47-52, 2001 .

NARVAI, P. C. et al. Cárie dentária no Brasil: declínio, iniquidade e exclusão social. Revista Panamericana de Salud Pública, Washington, DC, EUA, v. 19, n. 6, p. 385393, 2006.

SANTANA, V. G. D. et al. Análise da evolução do financiamento da assistência odontológica na média complexidade no município de Recife no período de 2000 a 2007. Cadernos Saúde Coletiva, Rio de Janeiro, v. 16, n. 3, p. 527-543, 2008.

SOARES, C. L. M.; PAIM, J. S. Aspectos críticos para a implementação da política de saúde bucal no Município de Salvador, Bahia, Brasil. Cadernos de Saúde Pública, Rio de Janeiro, v. 27, n. 5, p. 966-974, 2011.

SOUZA, L. F.; CHAVES, S. C. L. Política nacional de saúde bucal: acessibilidade e utilização de serviços Odontológicos especializados em um município de médio porte na Bahia. Revista Baiana de Saúde Pública, Salvador, v. 34, n. 2, p. 371-387, 2010.

\footnotetext{
Recebido para publicação em junho de 2015

Versão final em outubro de 2015

Conflito de interesses: inexistente

Suporte financeiro: Conselho Nacional de Desenvolvimento

Científico e Tecnológico ( $\mathrm{CNPq}$ ) e Fundação de Apoio à Pesquisa

do Estado da Paraíba (Fapesq), processo 092/2010, projeto PPSUS
} 